ARTICLE

https://doi.org/10.1038/s41467-019-09011-5

\title{
Modulation of recombination zone position for quasi-two-dimensional blue perovskite light-emitting diodes with efficiency exceeding 5\%
}

\author{
Zhenchao Li ${ }^{1}$, Ziming Chen ${ }^{1}$, Yongchao Yang ${ }^{1}$, Qifan Xue ${ }^{1}$, Hin-Lap Yip ${ }^{1} \&$ Yong Cao ${ }^{1}$
}

In recent years, substantial progress has been made in developing perovskite light-emitting diodes with near-infrared, red and green emissions and over 20\% external quantum efficiency. However, the development of perovskite light-emitting diodes with blue emission remains a great challenge, which retards further development of full-color displays and white-light illumination based on perovskite emissive materials. Here, firstly, through composition and dimensional engineering, we prepare quasi-two-dimensional perovskite thin films with improved blue emission, taking advantages of reduced trap density and enhanced photoluminescence quantum yield. Secondly, we find a vertically non-uniform distribution of perovskite crystals in the PEDOT:PSS/perovskite hybrid film. Through modulating the position of the recombination zone, we activate the majority of quasi-two-dimensional perovskite crystals, and thus demonstrate the most efficient blue perovskite light-emitting diode to date with emission peak at $480 \mathrm{~nm}$, record luminance of $3780 \mathrm{~cd} \mathrm{~m}^{-2}$ and record external quantum efficiency of $5.7 \%$.

\footnotetext{
${ }^{1}$ State Key Laboratory of Luminescent Materials and Devices, Institute of Polymer Optoelectronic Materials and Devices, School of Materials Science and Engineering, South China University of Technology, 381 Wushan Road, 510640 Guangzhou, China. These authors contributed equally: Zhenchao Li, Ziming Chen. Correspondence and requests for materials should be addressed to H.-L.Y. (email: msangusyip@scut.edu.cn)
} 
$\mathrm{n}$ recent years, metal halide perovskites have shown promising potential in a series of optoelectronic applications such as solar cells, light-emitting diodes (LEDs), photodetectors, and lasers $^{1-4}$. The booming research on metal halide perovskites can be attributed to their superior optoelectronic properties such as high absorption coefficient, long charge-carrier diffusion length, high photoluminescence quantum yield (PLQY), and high defect tolerance ${ }^{5-8}$. In particular, owing to the nature of their facile color tunability, solution processability, and sharp emission, metal halide perovskites have been successfully applied in LEDs, extending the family of emitters ${ }^{9-15}$. Substantial progress has been made over the past several years in perovskite LEDs by modulating the microstructures of the perovskite emitter, suppressing surface defects, and utilizing dimensional engineering. Based on these strategies, perovskite LEDs with near-infrared, red, and green emissions have reached over $20 \%$ external quantum efficiency $(\mathrm{EQE})^{16-18}$. However, compared with the efficient near-infrared, red, and green perovskite LEDs, blue perovskite LEDs currently still suffer from inferior performance. Improving performance remains a great challenge and retards the development of possible applications such as full-color displays and white-light illumination based on perovskite LEDs. Therefore, a breakthrough in thin film perovskite LEDs with blue emission is an urgent target in this field.

As blue emissions are generally considered to be in the range of $\sim 450-500 \mathrm{~nm}$, from deep blue to sky blue, in perovskite thin film emitters, there are typically two approaches that can achieve emission in this range: dimensional engineering and composition engineering. In terms of dimensional engineering, a hypsochromic shift of emission can be obtained by quantum confinement effect when reducing the dimension of perovskite from three dimensions to two dimensions. Generally, the blue photoluminescence (PL) and electroluminescence (EL) of perovskite thin films and LEDs can be achieved by forming quasi-twodimensional (quasi-2D) perovskite from a three-dimensional (3D) bulk perovskite. In our previous work, we incorporated a small molecule of 2-phenoxyethylamine into $\mathrm{CH}_{3} \mathrm{NH}_{3} \mathrm{PbBr}_{3}$ to form 2D perovskite to blue shift the EL from 532 to $494 \mathrm{~nm}$ and $462 \mathrm{~nm}$ to achieve the sky-blue and deep-blue perovskite LEDs, respectively ${ }^{19}$. A similar strategy was applied by using ethylammonium bromide as the ligand to form 2D perovskite thin films, which yielded a decent EQE of $2.6 \%$ with EL peaks of 473 and $485 \mathrm{~nm}^{20}$. To the best of our knowledge, this performance is the leading result among all of the published data. However, the relatively poor thermal stability and ion migration problems existing in organic-inorganic hybrid perovskite are detrimental to device stability, particularly in blue perovskite LEDs, which require a higher working voltage and hence stronger electric field for operation ${ }^{21,22}$. Therefore, a much more stable perovskite system is required for blue perovskite LEDs. Among the perovskite family, an all-inorganic perovskite system obtains much better structural and thermal stability than an organic-inorganic hybrid system ${ }^{23,24}$. Hence, it is possible to achieve a more stable blue perovskite LED by forming a quasi-2D perovskite from a green-emissive all-inorganic 3D perovskite of $\mathrm{CsPbBr}_{3}$. According to the previous reports and our own case (Supplementary Figure 1a), the formation of quasi-2D perovskite could indeed blue shift the PL from the green to blue region by the quantum confinement effect ${ }^{19,25}$. Unfortunately, in the case of perovskite LEDs based on these quasi-2D perovskite films, all the high energy blue emissions diminished and only the green-emissive EL was detected (Supplementary Figure 1b), and similar phenomenon was also observed in other report ${ }^{25}$. We attribute this phenomenon to the highly efficient energy transfer and/or charge transfer from larger bandgap perovskite to the smallest bandgap perovskite in such an all-inorganic quasi-2D perovskite system, as has also been observed in other reports and was used as a key strategy to improve the emission property of small bandgap perovskites ${ }^{26,27}$. The failure in achieving blue perovskite LED by forming quasi-2D perovskite based on a $\mathrm{CsPbBr}_{3}$ system prompts us to enlarge the bandgap of the $3 \mathrm{D}$ component in quasi-2D perovskite films to obtain a blue EL regardless of the presence of highly efficient energy transfer and/or charge transfer from the $2 \mathrm{D}$ to $3 \mathrm{D}$ component.

Composition engineering on halide anions is a proven strategy to tune the bandgap of $3 \mathrm{D}$ perovskite. It is well-known that partially replacing $\mathrm{Br}$ with $\mathrm{Cl}$ in an $\mathrm{AMBr}_{3}$ perovskite system (where A represents monovalent cations and $\mathrm{M}$ represents metal cations) can enlarge the bandgap of perovskite and hence blue shift its emission from green to blue ${ }^{28}$. Here we show the perovskite of $\mathrm{CsPbCl}_{0.9} \mathrm{Br}_{2.1}$ with emission at $484 \mathrm{~nm}$ in the blue region through composition engineering by incorporating $\mathrm{Cl}$ into $\mathrm{CsPbBr}$ lattice. Based on such a $3 \mathrm{D}$ perovskite system, phenylethylammonium bromide (PEABr) is subsequently introduced into the $\mathrm{CsPbCl}{ }_{0.9} \mathrm{Br}_{2.1}$ perovskite to form a quasi-2D perovskite thin film. We find that the PEABr can efficiently passivate the traps in perovskite thin films and dramatically improve their PLQYs from $0.15 \%$ to a maximum of $27 \%$. Moreover, we observe a vertically non-uniform distribution of perovskite crystals in the Poly(3,4-ethylenedioxythiophene):poly(styrene sulfonate) (PEDOT:PSS)/perovskite hybrid film in which the majority of perovskite crystals disperse at the top of the film. On the basis of the recombination zone control in this hybrid layer, we activate most of the perovskite emitters and thus successfully demonstrate a highly efficient blue perovskite LED with maximum EQE of $5.7 \%$ and maximum luminance of $3780 \mathrm{~cd} \mathrm{~m}^{-2}$, which, to our best knowledge, is a record performance for perovskite LEDs with blue emission.

\section{Results}

Optical properties of composition-engineered perovskites. Typically, all inorganic $\mathrm{Cs} \mathrm{PbBr}_{3}$ perovskite, which emits green light, is composed of cesium bromide $(\mathrm{CsBr})$ and lead bromide $\left(\mathrm{PbBr}_{2}\right)$. As previously mentioned, the incorporation of $\mathrm{Cl}$ into the $\mathrm{CsPBr}_{3}$ lattice makes possible the shift in emission from green to blue. However, the extremely poor solubility of $\mathrm{CsPbCl}$ (which is composed of cesium chloride $(\mathrm{CsCl})$ and $\left.\mathrm{PbCl}_{2}\right)$ in dimethyl sulfoxide (DMSO) limits the $\mathrm{Cl}$ concentration in the $\mathrm{CsPCl}_{x} \mathrm{Br}_{3-x}$ precursor solution and hence limits the degree of hypsochromic shift of emission. Compared to $\mathrm{PbCl}_{2}, \mathrm{CsCl}$ has a better solubility in DMSO, which can be applied as one of the precursor materials for $\mathrm{CsPCl}_{x} \mathrm{Br}_{3-x}$ to enhance its $\mathrm{Cl}$ concentration. Therefore, $\mathrm{CsCl}, \mathrm{CsBr}$, and $\mathrm{PbBr}_{2}$ are used to form $\mathrm{CsPbCl}_{x} \mathrm{Br}_{3-x}$ in our case. We studied the different molar ratios between $\mathrm{CsCl}$ and $\mathrm{CsBr}$ and the total molar ratio between $(\mathrm{CsCl}+\mathrm{CsBr})$ and $\mathrm{PbBr}_{2}$ is fixed to a $1: 1$ ratio in $\mathrm{Cs} \mathrm{PbCl}_{x} \mathrm{Br}_{3-x}$ precursor solution. According to Fig. 1, as we increased the $\mathrm{Cl}$ concentration in $\mathrm{CsPbCl}_{x} \mathrm{Br}_{3-x}$ by carefully increasing the amount of $\mathrm{CsCl}$, a gradual hypsochromic shift of absorption edges from green to blue was observed, illustrating that we could precisely modulate the emission color for $\mathrm{CsPCl}_{x} \mathrm{Br}_{3-x}$ perovskite. When the ratio of $\mathrm{CsCl}$ to $\mathrm{CsBr}$ reached 9:1, the absorption peak of $\mathrm{CsPbCl}_{0.9} \mathrm{Br}_{2.1}$ perovskite appeared at $478 \mathrm{~nm}$ in the blue region. However, when we further increased the ratio to $1: 0$, precipitation occurred in the precursor solution (Supplementary Figure 2), suggesting that the precursor solution reached a solubility limit and became unsuitable for further processing into thin films. According to Fig. 1b, a similar trend was also observed in the PL spectra. When we increased the $\mathrm{Cl}$ concentration in the $\mathrm{Cs} \mathrm{PbCl}_{x} \mathrm{Br}_{3-x}$ lattice, a gradual hypsochromic shift of emission was obtained, and the bluest emission, which 
a

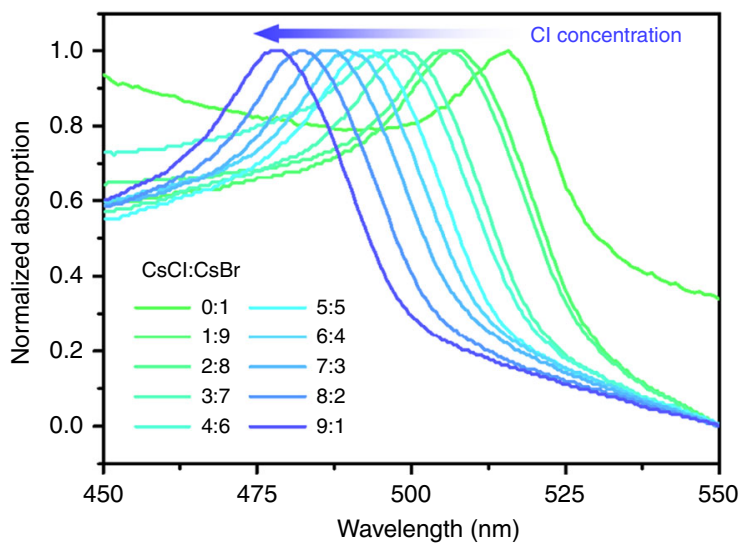

C

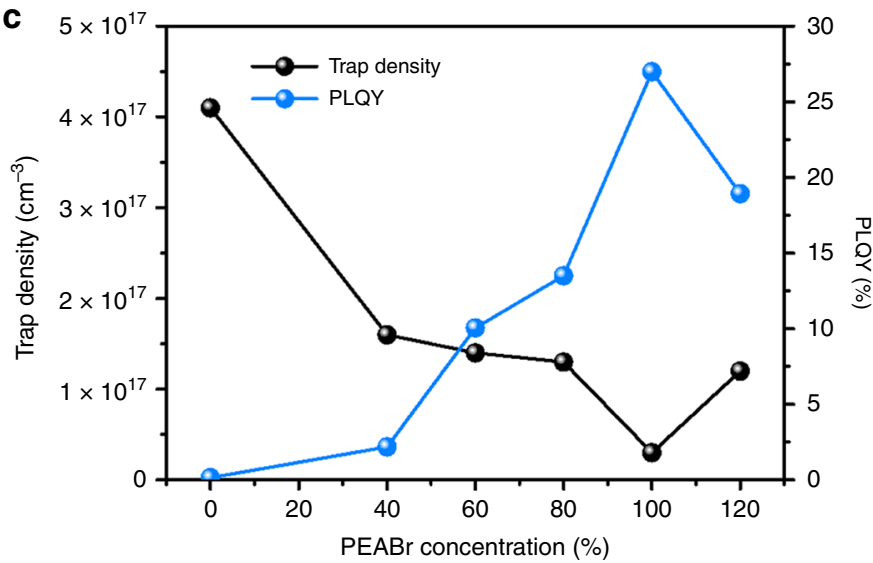

b

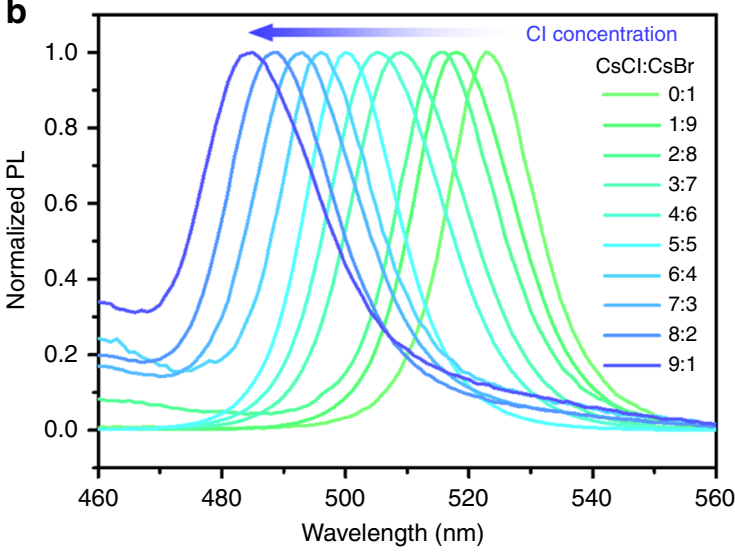

d

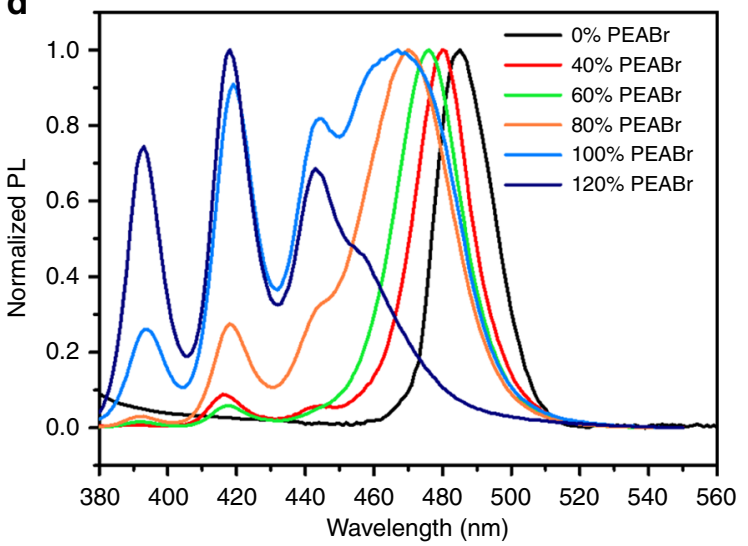

Fig. 1 Composition engineering for efficient blue emission of perovskite. a Normalized absorption of $\mathrm{CsPbCl}_{x} \mathrm{Br}_{3}-x$ thin films with different molar ratios of cesium chloride $(\mathrm{CsCl})$ to cesium bromide $(\mathrm{CsBr})$. The results are normalized by the intensity of their corresponding excitonic absorption peaks. b Photoluminescence (PL) spectra of $\mathrm{CsPbCl}_{x} \mathrm{Br}_{3-x}$ thin films with different molar ratios of $\mathrm{CsCl}$ to $\mathrm{CsBr}$. c Trap densities and photoluminescence quantum yields ( $\mathrm{PLQYs}$ ) of $\mathrm{CsPbCl}_{0.9} \mathrm{Br}_{2.1}$ thin films with different ratios of phenylethylammonium bromide ( $\mathrm{PEABr}$ ). The trap density was calculated based on the equation $N_{t}=2 \varepsilon_{0} \varepsilon_{r} V_{T F L} /\left(q L^{2}\right)$, and a detailed discussion can be found in Supplementary Figures 3 and 4 . d PL spectra of $C s P b l_{0.9} B_{2.1}$ thin films with different ratios of $\mathrm{PEABr}$

was achieved by the $\mathrm{CsPbCl}{ }_{0.9} \mathrm{Br}_{2.1}$ perovskite, was located at the wavelength of $484 \mathrm{~nm}$ in the blue region. Therefore, the composition of $\mathrm{CsPbCl}_{0.9} \mathrm{Br}_{2.1}$ was selected as the $3 \mathrm{D}$ perovskite system for our subsequent study aiming to realize the target of an efficient blue perovskite LED.

In metal halide perovskites, trap-assisted recombination, which relates to trap density in perovskite thin films, is a typically nonradiative recombination ${ }^{2}$. According to Fig. 1c, the trap density in a pristine $\mathrm{CsPCCl}_{0.9} \mathrm{Br}_{2.1}$ perovskite thin film is around $4.1 \times$ $10^{17} \mathrm{~cm}^{-3}$, causing a low PLQY of around $0.15 \%$. Noticing that the charge-carrier density in a working LED is generally of the order of $<10^{16} \mathrm{~cm}^{-3}$, it can be expected that such high trap density in pristine $\mathrm{CsPbCl}_{0.9} \mathrm{Br}_{2.1}$ perovskite thin film will probably lead to inferior performance of the corresponding LED device ${ }^{29}$. Therefore, the suppression of trap-assisted recombination by reducing the trap density is critical to achieving a higher PLQY and better device performance. Recent reports prove that $2 \mathrm{D}$ perovskite obtains a much lower trap density compared with its $3 \mathrm{D}$ counterpart because the organic ligands (such as 2phenoxyethylamine and butylammonium) generally passivate surface traps of perovskite ${ }^{19,30}$. With this inspiration, we used $\mathrm{PEABr}$ to form $2 \mathrm{D}$ perovskite and passivated its surface traps based on the $\mathrm{Cs} \mathrm{PbCl}_{0.9} \mathrm{Br}_{2.1}$ system. Encouragingly, the trap density of perovskite thin films indeed dramatically reduced from $\sim 4.1 \times 10^{17}$ to $3.0 \times 10^{16} \mathrm{~cm}^{-3}$ as the ratio of PEABr was increased from 0 to $100 \%$ (Supplementary Figures 3 and 4).
However, a further increase in PEABr concentration to $120 \%$ resulted in an increase of trap density to around $1.2 \times 10^{17} \mathrm{~cm}^{-3}$. This result was attributed to the reduced crystal quality in perovskite films (which will be discussed later). In addition, we performed PL lifetime measurement to cross-check the trap passivation effect. The PL lifetimes of perovskite films increased as the increase of PEABr ratios (Supplementary Figure 5), which suggested that the surface trap states of perovskite had been well passivated by the PEABr molecules. Furthermore, from Fig. 1c, we can observe that the PLQY is increased with reducing the trap density of the perovskite film. The PLQYs of the perovskite thin films dramatically increase from 0.15 to $27 \%$ with the increase of $\mathrm{PEABr}$ ratios from 0 to $100 \%$, and it decreases again to $18.9 \%$ when an overload of $120 \%$ PEABr was added into the $\mathrm{CsPbCl}{ }_{0.9} \mathrm{Br}_{2.1}$ system. The relationship between trap densities and PLQYs of perovskite thin films shows that trap-assisted recombination dominates the non-radiative recombination, and trap reduction in such mixed-halide perovskites is critical to achieving efficient blue emission.

We also recorded the PL spectra of $\mathrm{CsPbCl}_{0.9} \mathrm{Br}_{2.1}$ thin films with different ratios of $\mathrm{PEABr}$ to reveal their emission colors. Interestingly, as the PEABr ratio increased, the emission peaks gradually blue shifted, and several specific peaks appeared in the deep-blue region. Similar to the $\mathrm{CsPbBr}$ system discussed previously, the presence of excitonic emission peaks at $393,418,444$, and $457 \mathrm{~nm}$ reflected the formation of $2 \mathrm{D}$ 
$\mathrm{PEA}_{2} \mathrm{Cs}_{n-1} \mathrm{~Pb}_{n}\left(\mathrm{Cl}_{x} \mathrm{Br}_{1-x}\right)_{3 n+1}$ perovskite (corresponding to $n=1,2,3$, and 4 , respectively). Therefore, $2 \mathrm{D}$ perovskites with $n=1,2$, and 3 coexisted in $\mathrm{CsPbCl}_{0.9} \mathrm{Br}_{2.1}$ thin films with $40 \%$ $\mathrm{PEABr}$, while the $\mathrm{CsPbCl}_{0.9} \mathrm{Br}_{2.1}$ thin films with $60 \%, 80 \%$, $100 \%$, and $120 \% \mathrm{PEABr}$ contained $2 \mathrm{D}$ perovskites with $n$ values of $1,2,3$, and 4 . In addition, the resultant films are quasi-2D perovskite films composed of mixture of both 2D and 3D phases as indicated by the coexistence of characteristic emission at lower energy from the 3D component.

Nanostructure and morphology of perovskite thin films. To further confirm the coexistence of $2 \mathrm{D}$ and $3 \mathrm{D}$ perovskites in the film, we used X-ray diffraction (XRD) measurement to study the structural property of the perovskite films. According to Fig. 2a, in pristine $\mathrm{Cs} \mathrm{PbCl}_{0.9} \mathrm{Br}_{2.1}$ thin film, a main peak at $2 \theta$ of $15.4^{\circ}$ appeared, corresponding to the (100) plane of $3 \mathrm{D}$ perovskite. When we added $40 \%$ PEABr into $\mathrm{CsPbCl}_{0.9} \mathrm{Br}_{2.1}$ thin films, a specific peak at $2 \theta$ of $5.3^{\circ}$ appeared, reflecting the formation of $n=12 \mathrm{D}$ perovskite ${ }^{31}$. When we further increased the PEABr ratio to over $60 \%$, another specific peak at $2 \theta$ of $3.9^{\circ}$ appeared, and we accordingly assigned it to the $n=22 \mathrm{D}$ perovskite. According to previous report that when PEABr was used as the organic spacer to form $2 \mathrm{D}$ perovskite based on $\mathrm{Cs}_{x} \mathrm{MA}_{1-x} \mathrm{PbBr}_{3}$ system, the $n=12 \mathrm{D}$ perovskite obtained the lowest formation energy than that in the $2 \mathrm{D}$ perovskites with higher $n$, which might explain the formation of $n=12 \mathrm{D}$ perovskite even though the $\mathrm{PEABr}$ ratio was low ${ }^{32}$. In addition, although we dramatically increased the PEABr ratio from 0 to $120 \%$, the XRD pattern from $3 \mathrm{D}$ perovskite was always present, also suggesting that the film we obtained was a quasi-2D perovskite film that combined with $2 \mathrm{D}$ and $3 \mathrm{D}$ perovskites. It is noteworthy that based on PL measurement, several specific emission peaks from different types of $2 \mathrm{D}$ perovskite $(n=1,2,3$, and 4$)$ appeared, whereas only $n=1$ and/ or $n=2$ perovskites were detected in the XRD measurements. We attributed this mismatch to two possible reasons: first, the orientations of $2 \mathrm{D}$ perovskite crystals with higher $n$ value are not in out-of-plane direction, which become undetectable in the onedimensional XRD measurement; second, the non-periodic alignment of these $2 \mathrm{D}$ perovskite crystals with higher $n$ value caused the absence of Bragg diffraction and hence the absence of an XRD signal ${ }^{33}$.

The morphology of perovskite thin films is another important factor governing the final performance of perovskite LEDs. Therefore, we used a scanning electron microscope (SEM) to characterize the morphology of perovskite thin films with different ratios of $\mathrm{PEABr}$. Figure $2 \mathrm{~b}$ shows that the pristine $\mathrm{CsPbCl}_{0.9} \mathrm{Br}_{2.1}$ film has a poor morphology with a large number of pinholes. However, after the introduction of $\mathrm{PEABr}$, perovskite films with better morphology and coverage were obtained. As the $\mathrm{PEABr}$ ratio increased from 40 to $100 \%$, the perovskite crystal became more and more regular in shape, suggesting improved crystal quality of the perovskite thin film. Particularly in the $100 \%$ $\mathrm{PEABr}$ case, a perovskite film with isolated and regular perovskite small crystals was obtained, reflecting the formation of a highquality perovskite film that correlated well with its low trap density and high PLQY. However, when we further increased the PEABr ratio to $120 \%$, the perovskite crystal returned to an irregular shape, resulting in a perovskite film with poorer crystal quality. It is likely that too much $\mathrm{PEABr}$ suppressed the formation of perovskite crystals, with a similar phenomenon also being observed when perovskite was mixed with other aminecontaining materials ${ }^{16,34}$.

Device structure and performance. To evaluate the EL properties of $\mathrm{CsPbCl}_{0.9} \mathrm{Br}_{2.1}$ thin films with different ratios of $\mathrm{PEABr}$, we fabricated perovskite LEDs based on the device architecture of indium tin oxide (ITO)/PEDOT:PSS/perovskite/1, 3, 5-tris $(\mathrm{N}$ phenylbenzimiazole-2-yl)benzene (TPBi)/lithium fluoride (LiF)/ $\mathrm{Al}$, as shown in Fig. 3a. Here, PEDOT:PSS (CH 8000, $45 \mathrm{~nm}$ thick) with a deep work function of $-5.15 \mathrm{eV}$ was used as the hole injection layer ${ }^{35}$. The valance band of perovskite was confirmed by ultraviolet photoelectron spectroscopy (UPS) as shown (Supplementary Figure 6) and the energy diagram of the whole device is presented in Fig. 3a. We also summarize the device performance in Fig. 3b, $\mathrm{c}$ and Table 1 . In the pristine $\mathrm{CsPbCl}{ }_{0.9} \mathrm{Br}_{2.1}$ perovskite LED, an inferior EQE of only $0.05 \%$ was achieved. When we increased the PEABr ratio in $\mathrm{CsPCl}_{0.9} \mathrm{Br}_{2.1}$ perovskite thin films from 0 to $100 \%$, the device performance improved dramatically, and the best device reached a high EQE of $3.6 \%$. However, further increasing the $\mathrm{PEABr}$ ratio to $120 \%$ reduced the EQE and luminance. There are three potential reasons for these results. First, the trend of EQE change with increasing PEABr ratios is similar to that of PLQYs of perovskite films, suggesting that the EL property of perovskite LEDs is governed by the PL property of the corresponding perovskite thin films. Second, the EL property also strongly related to the trap densities of perovskite films as higher trap density could result in more severe trapping of injected charge carriers and hence cause a more severe a

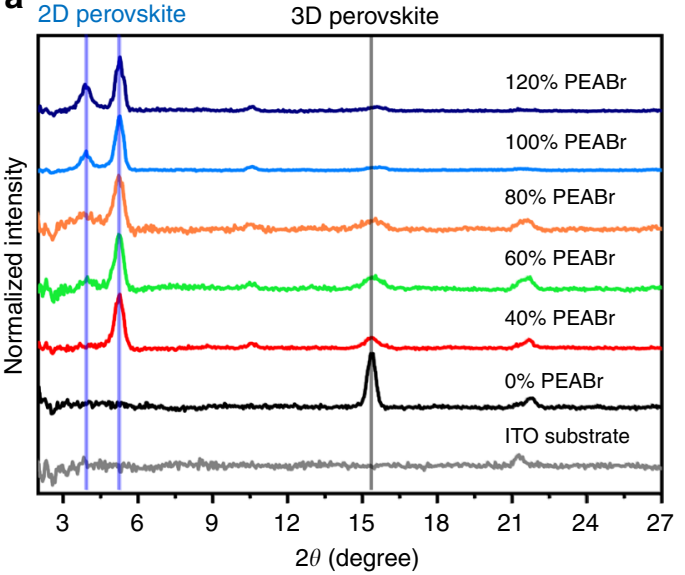

b

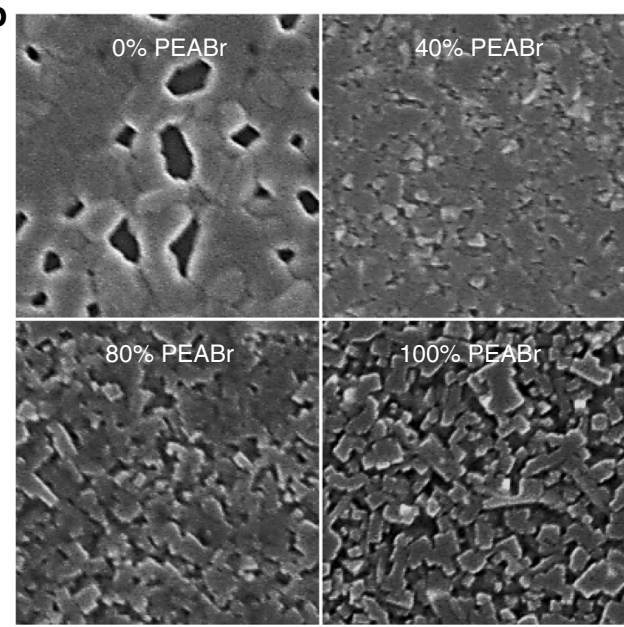

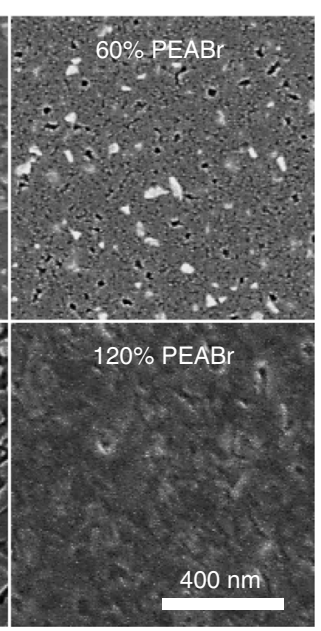

Fig. 2 Crystal structure and morphology of $\mathrm{CsPbCl}_{0.9} \mathrm{Br}_{2.1}$ thin films with different ratios of phenylethylammonium bromide ( $\mathrm{PEABr}$ ). a X-ray diffraction (XRD) patterns and $\mathbf{b}$ top-view scanning electron microscope (SEM) images at a size of $1 \times 1 \mu \mathrm{m}^{2}$ 
a
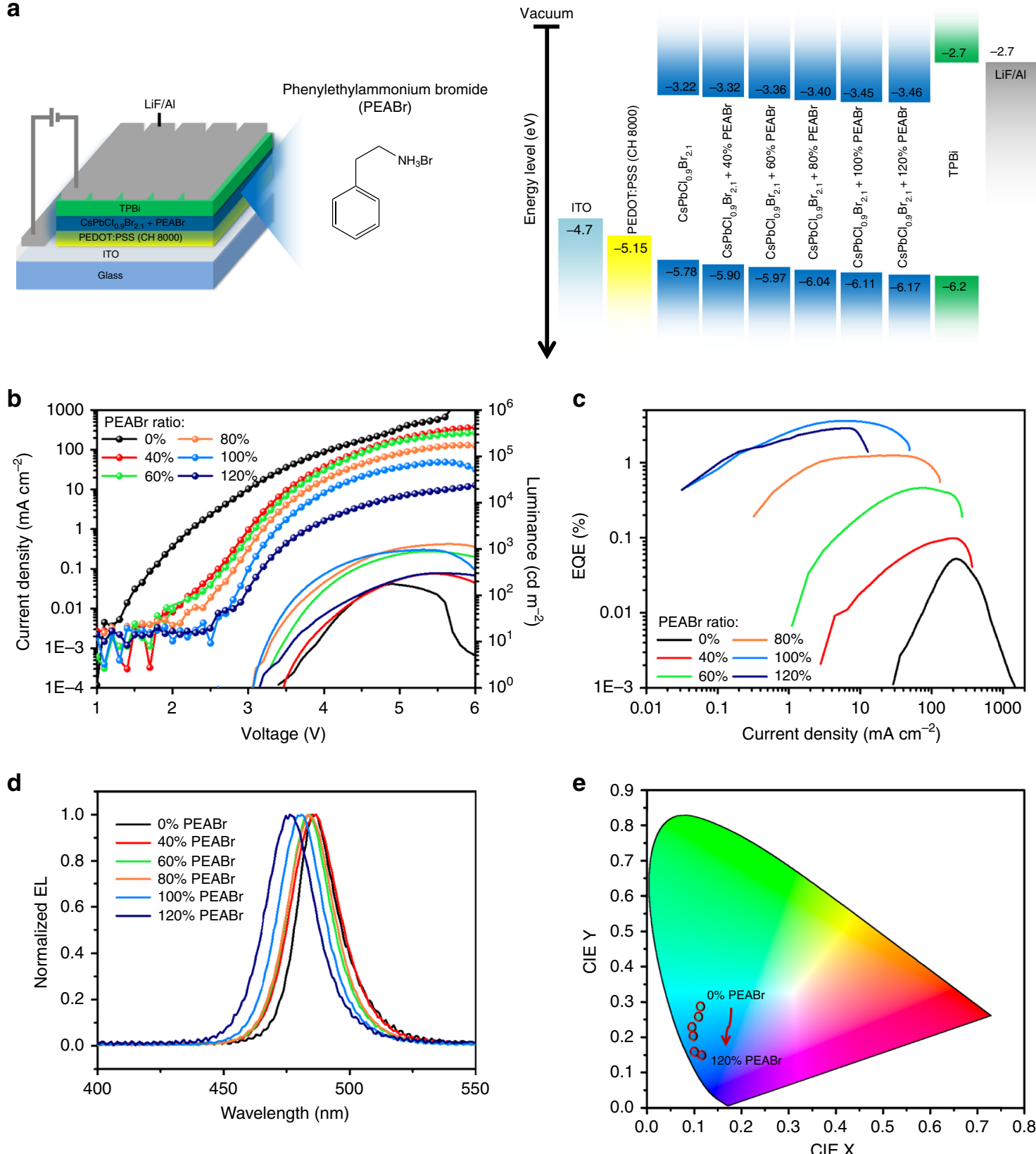

Fig. 3 Performance of $\mathrm{CsPbCl}_{0.9} \mathrm{Br}_{21}$ perovskite light-emitting diodes (LEDs) with different ratios of phenylethylammonium bromide (PEABr). a Perovskite LED architecture and energy level diagram for different layers of the device. $\mathbf{b}$ Characterization of current density and luminance versus voltage. The solid lines and lines with dots correspond to the luminance and the current density, respectively. c Characterization of external quantum efficiency (EQE) versus current density. d Electroluminescence (EL) spectra of perovskite LEDs. e Commission Internationale de I'Eclairage (CIE) values of the EL spectra of perovskite LEDs

Table 1 Summary of $\mathrm{CsPbCl}_{0.9} \mathrm{Br}_{2.1}$ perovskite LEDs with different ratios of $\mathrm{PEABr}$

\begin{tabular}{|c|c|c|c|c|c|c|c|}
\hline PEABr ratio & $V_{T}(V)$ & EL peak (nm) & Max. L. $\left(\mathrm{cd} \mathrm{m}^{-2}\right)$ & Max. CE $\left(\mathrm{cd} \mathrm{A}^{-1}\right)$ & Max. EQE (\%) & FWHM (nm) & CIE \\
\hline $0 \%$ & 3.5 & 487 & 177 & 0.07 & 0.05 & 20 & $(0.112,0.289)$ \\
\hline $40 \%$ & 3.5 & 485 & 293 & 0.12 & 0.10 & 23 & $(0.105,0.254)$ \\
\hline $60 \%$ & 3.3 & 484 & 892 & 0.52 & 0.46 & 21 & $(0.093,0.222)$ \\
\hline $80 \%$ & 3.2 & 483 & 1278 & 1.43 & 1.25 & 21 & $(0.096,0.204)$ \\
\hline $100 \%$ & 3.1 & 480 & 965 & 3.90 & 3.60 & 21 & $(0.101,0.155)$ \\
\hline $120 \%$ & 3.3 & 478 & 304 & 2.69 & 2.88 & 24 & $(0.113,0.149)$ \\
\hline
\end{tabular}

LED light-emitting diode, PEABr phenylethylammonium bromide, $V_{T}$ turn-on voltage, CE current efficiency, EQE external quantum efficiency, FWHM full-width at half-maximum, CIE Commission Internationale de l'Eclairage 
non-radiative trap-assisted recombination. Third, the pristine perovskite thin film suffered from poor morphology due to the large number of pinholes that caused a severe leakage current in the LED device, while better film coverage and crystal quality were obtained after the introduction of $\mathrm{PEABr}$ from 0 to $100 \%$, which also resulted in the suppression of leakage current in perovskite LEDs according to Fig. 3b. However, a further increase in the PEABr concentration to $120 \%$ led to a film with decreased crystal quality compared with the $100 \%$ PEABr case, causing a slightly drop in device performance.

Figure $3 \mathrm{~d}$ shows the EL spectra of the $\mathrm{Cs} \mathrm{PbCl}_{0.9} \mathrm{Br}_{2.1}$ perovskite LEDs with different ratios of $\mathrm{PEABr}$ and all the EL emissions obtained narrow full-widths at half-maximum (FWHMs) of $<25 \mathrm{~nm}$, resulting in the high color purity in Commission Internationale de l'Eclairage (CIE) coordinate as shown in Fig. 3e. Similar to the $\mathrm{CsPbr}_{3}$ system, although excitonic PL from 2D $\mathrm{PEA}_{2} \mathrm{Cs}_{n-1} \mathrm{~Pb}_{n}\left(\mathrm{Cl}_{x} \mathrm{Br}_{1-x}\right)_{3 n+1}$ perovskites was observed, only one single EL peak at the lowest energy belonging to the $3 \mathrm{D}$ perovskite was detected in all cases, suggesting that energy transfer and/or charge transfer from higher-energy $2 \mathrm{D}$ perovskite to lower-energy 3D perovskite is also highly efficient in such a quasi-2D system. In addition, a gradual blue shift of EL from 487 to $478 \mathrm{~nm}$ was observed as the PEABr ratio increased, which correlated well with the blue shift of PL spectra because of the gradual loss of the 3D nature of the perovskite film. With the blue shift of EL spectra, the corresponding CIE value also changed from the sky blue to blue region, suggesting the successful achievement of a blue-emissive perovskite LED.

Modulation of recombination zone position. Recently, several reports have shown that a vertically non-uniform distribution of perovskite nanostructure exists in quasi-2D perovskite thin films. Wang et al. $^{36}$ reported a vertical element distribution in the perovskite multi-quantum wells in which the $\mathrm{Pb}$ signal gradually decreased from the top to the bottom of the perovskite thin film, suggesting that the $3 \mathrm{D}$ perovskite was mainly located at the top of the perovskite film, whereas the $2 \mathrm{D}$ perovskite was enriched at the bottom. In addition, Shang et al. ${ }^{25}$ reported that only the PL from $3 \mathrm{D}$ perovskite could be detected under photoexcitation from the top side, whereas PL from the 2D perovskite appeared with photoexcitation from the bottom side, indirectly reflecting the vertical distribution of the perovskite nanostructure. According to these previous studies, it can be expected that the position of the recombination zone for the injected charge carriers is critical to achieving a highly efficient perovskite LED if a vertically nonuniform distribution of nanostructure is existed in the perovskite thin film. Therefore, we used a scanning transmission electron microscope to study the vertical morphology of the perovskite thin film to reveal the distribution of the perovskite. According to Fig. 4a, we obtained a PEDOT:PSS/perovskite hybrid film without a distinct interface, although both the PEDOT:PSS and perovskite films were deposited layer by layer. The majority of perovskite crystals dispersed at the top of the hybrid film, whereas some crystals were embedded within the PEDOT:PSS layer, leaving a perovskite capping layer above the PEDOT:PSS film and resulting in a vertically non-uniform distribution of the perovskite crystals. We attributed this result to the rough PEDOT: PSS films regardless of the film thickness (Supplementary Figure 7), which allowed a few perovskite crystals filling into the voids and being embedded by the PEDOT:PSS layer.

In addition, we also used an energy-dispersive spectrometer (EDS) to further unveil the elemental distribution of perovskite crystals. Figure $4 \mathrm{a}$ shows the distribution of the $\mathrm{Cs}, \mathrm{Pb}, \mathrm{Br}$, and $\mathrm{Cl}$ signals and their integrated signals as a function of depth. It is obvious to find that most of the elements present at the top of the film, showing a similar distribution without distinct compositional difference. In addition, the integrated $\mathrm{Cs}, \mathrm{Pb}, \mathrm{Br}$, and $\mathrm{Cl}$ signals perfectly overlap with each other as a function of depth, illustrating that all of the perovskite crystals (from top to bottom) are in a similar stoichiometric ratio. This result provides strong evidence that every single perovskite crystal has a similar quasi-

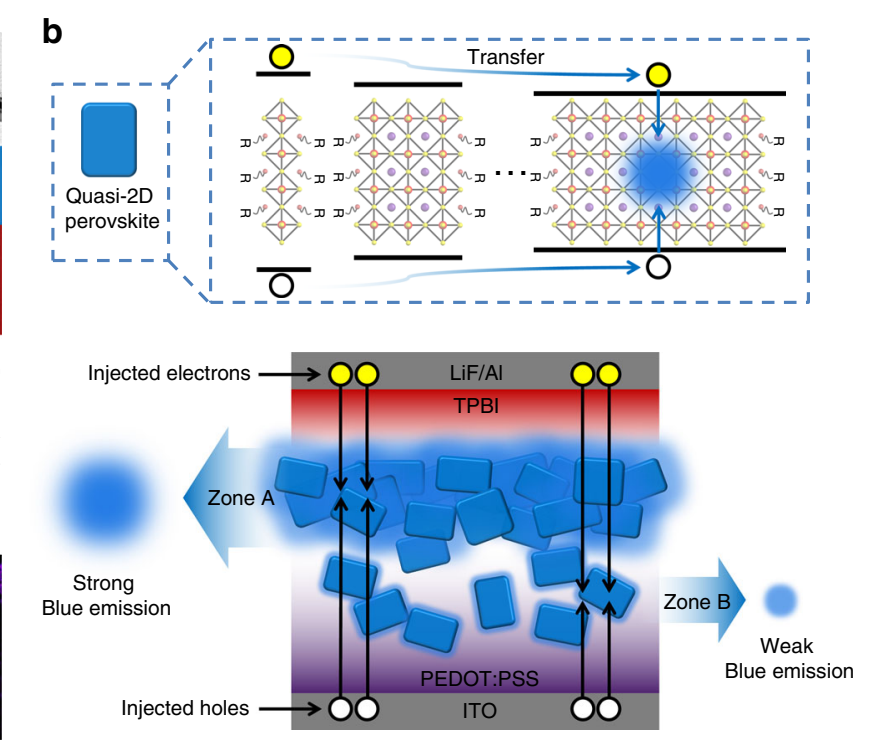

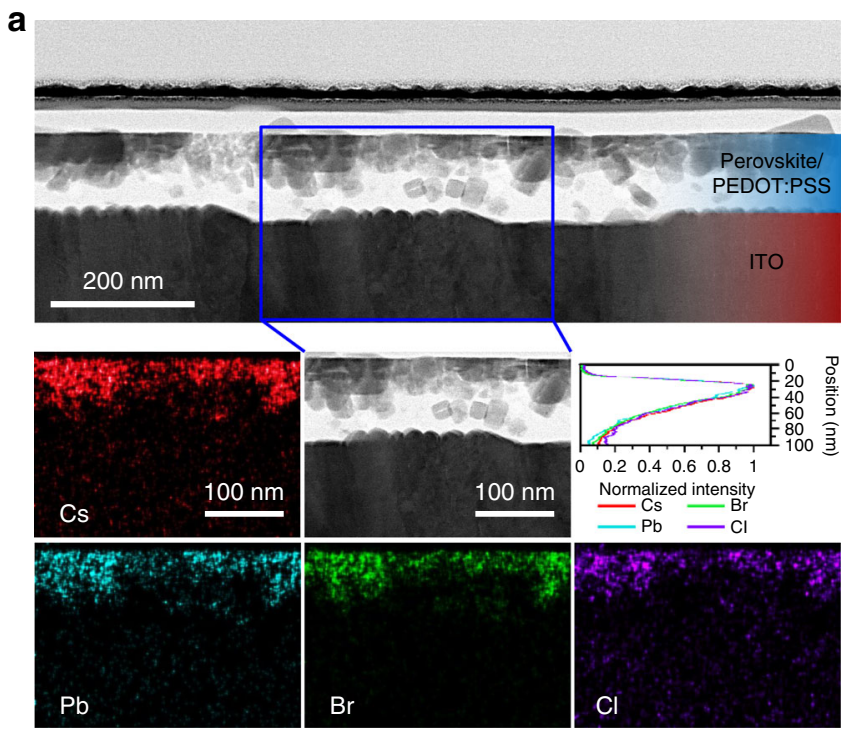

Fig. 4 Vertical morphology of the perovskite thin film and schematic diagram of the modulation of recombination zone position. a Cross-section scanning transmission electron microscope (STEM) image of a indium tin oxide (ITO)/PEDOT:PSS/perovskite film. Energy-dispersive spectrometer (EDS) measurement was also used at the region marked with the blue lines to illustrate the elemental distribution of $\mathrm{Cs}, \mathrm{Pb}, \mathrm{Br}$, and $\mathrm{Cl}$. The position versus normalized intensity curves illustrate the integrated intensity of the $\mathrm{Cs}, \mathrm{Pb}, \mathrm{Br}$, and $\mathrm{Cl}$ signals as a function of depth. The perfect overlapping suggests the homogenous distribution of all four signals in perovskite crystals. b The top schematic diagram shows that each perovskite crystal is in a quasi-2D structure, and energy transfer and/or charge transfer occurs after the injection of electrons and holes. The bottom schematic diagram shows that as most of the perovskite crystals disperse at the top of the film, if the injected electrons and holes can recombine in Zone A, a strong blue emission can be obtained; however, if the injected electrons and holes can only recombine in Zone B, the film shows a weak blue emission 

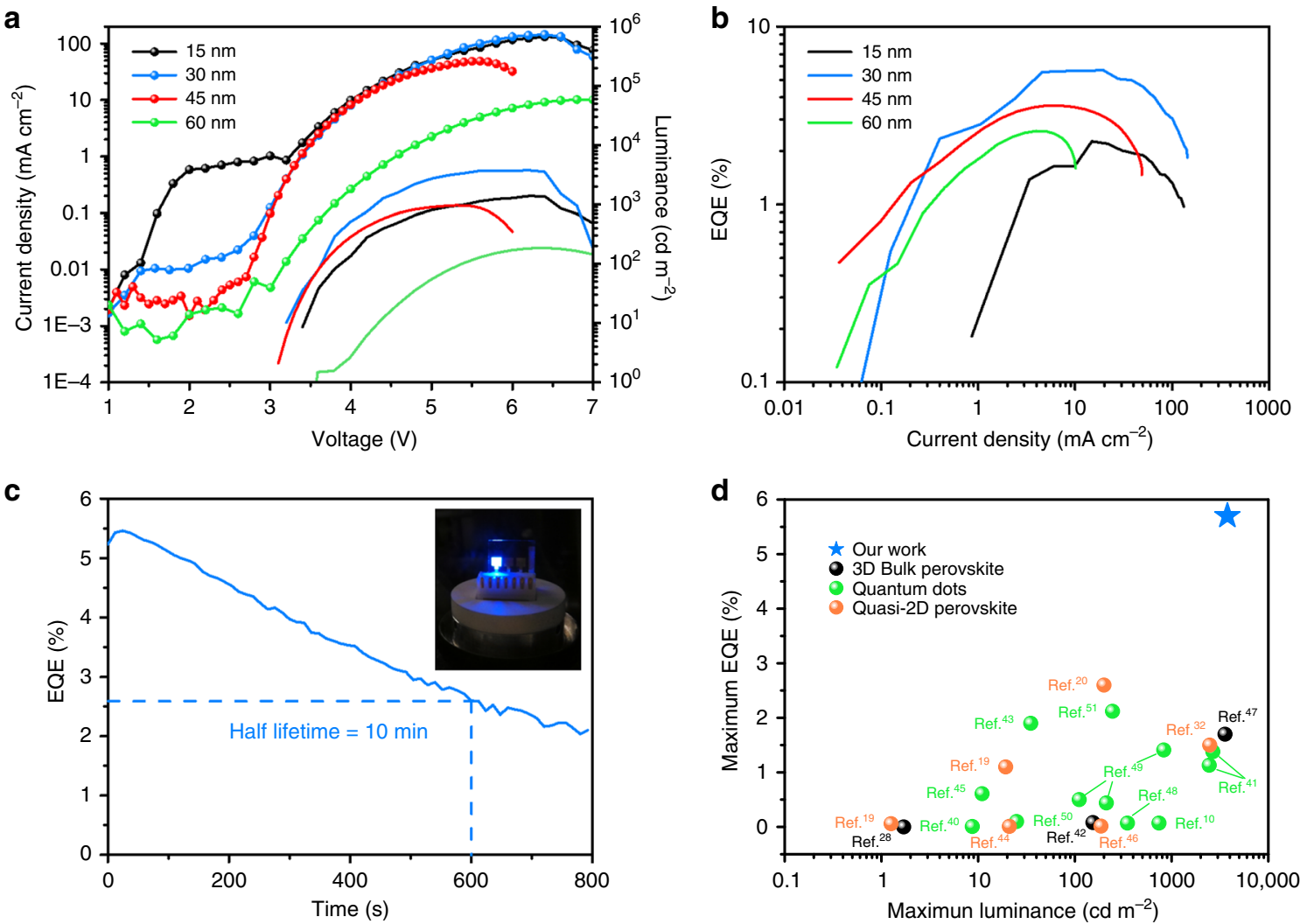

Fig. 5 Performance of perovskite light-emitting diode (LEDs) (100\% phenylethylammonium bromide (PEABr) case) with different PEDOT:PSS thicknesses. a Characterization of current density and luminance versus voltage. The solid lines and lines with dots correspond to luminance and current density, respectively. $\mathbf{b}$ Characterization of external quantum efficiency (EQE) versus current density. c Operating lifetime of a perovskite LED under a constant applied voltage of $4.4 \mathrm{~V}$ and a photo of a working perovskite LED. d Comparison of our work with the currently published results based on blue-emissive perovskite LEDs with emission in the range of $450-500 \mathrm{~nm}$

2D structure and that no obvious nanostructural distribution occurred in our case, which is different from that observed in previously mentioned reports ${ }^{25,36}$. Therefore, each perovskite crystal is a single emitter and by combining the EL spectra of the CsPbCl ${ }_{0.9} \mathrm{Br}_{2.1}$ perovskite LEDs with different ratios of $\mathrm{PEABr}$, we can conclude that in a working perovskite LED, highly efficient energy transfer and/or charge transfer occurs within those single quasi-2D perovskite crystals, as illustrated in the top schematic diagram in Fig. 4b. In addition, the observation of vertically nonuniform distribution of the perovskite crystals encouraged us to further modulate the position of the recombination zone in the device aiming to produce a more efficient perovskite LED.

The recombination zone is the region in which injected electrons and holes meet and recombine with each other. For LED applications, we expect that the recombination zone should be located at the emissive layer to convert electrical energy into light. In addition, the recombination zone width is also a critical parameter, and it is calculated to be ca. $5.5 \mathrm{~nm}$ in our best perovskite LED (Supplementary Note 1 and Supplementary Figure 8). As previously mentioned, the majority of emissive perovskite crystals dispersed on the top of the perovskite film (assigned Zone A in Fig. 4b), while a small number of perovskite crystals dispersed at the bottom of the perovskite film (assigned Zone B in Fig. 4b). Therefore, it should be much better for the injected electrons and holes to recombine in Zone A rather than in Zone B to activate the greatest number of perovskite crystals to realize a strong blue emission, as illustrated in the bottom schematic diagram of Fig. $4 \mathrm{~b}$.

Generally, the position of the recombination zone in the emissive layer can be simply controlled by the thickness of the hole and electron injection layers ${ }^{37-39}$. Considering perovskite LED is typically a hole-dominant device when using PEDOT:PSS and TPBi as the hole and electron injection layer, respectively, we focused our study on the thickness control of the PEDOT:PSS layer to obtain an optimized position of the recombination zone $^{35}$. We tuned the thickness of the PEDOT:PSS layer from 15 to $60 \mathrm{~nm}$ in the $\mathrm{CsPbCl}_{0.9} \mathrm{Br}_{2.1}$ perovskite LEDs with $100 \%$ PEABr, which has shown the best EQE in our study. According to Fig. 5a, b and Table 2, when the PEDOT:PSS layer was thick $(60 \mathrm{~nm})$, a relatively poor EQE of $2.6 \%$ was obtained in the perovskite LED because in a thick PEDOT:PSS case, the injected holes should take a relatively long time to pass through the thick PEDOT:PSS layer and subsequently inject into the valence band of perovskite, which results in a recombination zone position closer to Zone B. When we decreased the film thickness from 60 to $45 \mathrm{~nm}$ and $30 \mathrm{~nm}$, the EQE of the perovskite LEDs increased dramatically from $2.6 \%$ to $3.6 \%$ and $5.7 \%$, respectively. In such a case, the thinner PEDOT:PSS layer allowed the injected holes to pass through in a shorter time, resulting in a position shift of the recombination zone toward Zone $\mathrm{A}$, which the electrons can reach in a short time. As the position of the recombination zone gradually shifted from Zone $\mathrm{B}$ to Zone $\mathrm{A}$ as the PEDOT:PSS thickness decreased, more and more perovskite crystals were activated, leading to an increase in device performance. However, when we further decreased the PEDOT:PSS thickness to $15 \mathrm{~nm}$, the perovskite LED showed a relatively poor EQE of $2.2 \%$. This result can be attributed to the poor coverage of such an ultra-thin PEDOT:PSS film on ITO, which may allow the perovskite crystals to directly get contact with the bottom electrode and therefore led to a severe leakage current in the perovskite LED (as shown in 
Table 2 Performance of perovskite LEDs (100\% PEABr case) with different PEDOT:PSS thicknesses

\begin{tabular}{|c|c|c|c|c|c|c|c|}
\hline PEDOT:PSS Thickness & $V_{T}(V)$ & EL peak $(\mathrm{nm})$ & Max. L. $\left(c d \mathrm{~m}^{-2}\right)$ & Max. CE $\left(\mathrm{cd} \mathrm{A}^{-1}\right)$ & Max. EQE (\%) & FWHM (nm) & CIE \\
\hline $30 \mathrm{~nm}$ & 3.2 & 480 & 3780 & 6.1 & 5.7 & 21 & $(0.102,0.178)$ \\
\hline $60 \mathrm{~nm}$ & 3.6 & 480 & 178 & 2.6 & 2.6 & 22 & $(0.101,0.148)$ \\
\hline
\end{tabular}

LED light-emitting diode, PEDOT:PSS Poly(3,4-ethylenedioxythiophene):poly(styrene sulfonate), CE current efficiency, EQE external quantum efficiency, PEABr phenylethylammonium bromide

Fig. 5a) that degraded device performance. It is worth emphasizing that based on recombination zone modulation, our best blue perovskite LED achieved a record luminance of $3780 \mathrm{~cd} \mathrm{~m}^{-2}$ and a record EQE of $5.7 \%$, which is more than twice that of the currently published data on blue perovskite LEDs, as shown in Fig. 5d $\mathrm{d}^{10,19,20,28,32,40-51}$. Our devices also obtained excellent device reproducibility with average EQE of $4.5 \%$ (Supplementary Figure 9).

In addition, our devices presented decent spectrum stability with no shift of the EL spectrum observed when we increased the applied voltage of the LED from 3.6 to $5.6 \mathrm{~V}$ (Supplementary Figure 10a). However, when we further increased the applied voltage exceeding $6 \mathrm{~V}$, the EQE and luminance of the device started to drop, and the EL spectrum also red shifted slightly by around $8 \mathrm{~nm}$, which could be attributed to the phase separation within the perovskite film due to the migration of $\mathrm{Cl}^{-}$and $\mathrm{Br}^{-40}$. Moreover, the operating lifetime of the devices were also studied under a constant applied voltage of $4.4 \mathrm{~V}$, as shown in Fig. $5 \mathrm{c}$. Our device obtained a half lifetime of around $10 \mathrm{~min}$, which is one of the best results among the reported lifetimes of blueemitting perovskite LEDs, as summarized in Supplementary Table 1. In addition, the EL spectrum of the device was tracked during the operation. As shown in Supplementary Figure 10b, the initial emission peak was located at $480 \mathrm{~nm}$ and no spectrum shift was observed at the initial $1 \mathrm{~min}$, followed by a slight red shift of $3 \mathrm{~nm}$ in the next $3 \mathrm{~min}$. During the operating time from 3 to 9 min, the emission peak continuously shifted from 483 to $488 \mathrm{~nm}$ and eventually pined at $488 \mathrm{~nm}$ for the rest of testing time. We attributed this slight red shift of spectrum to the possible phase separation occurred in the mixed-halide perovskite system. It is worth noting that other degradation processes may also occur, which requires a further investigation in the future to fully unveil the degradation mechanism.

\section{Discussion}

Here, we demonstrate a highly efficient blue perovskite LED by combining the strategies of composition engineering in quasi-2D perovskite crystals and modulation of the recombination zone position. In terms of composition engineering, $\mathrm{CsCl}, \mathrm{CsBr}$, and $\mathrm{PbBr}_{2}$ are used to prepare the precursor solution, and the molar ratio of $\mathrm{CsCl}: \mathrm{CsBr}: \mathrm{PbBr}_{2}$ of $9: 1: 10$ is used to form the blueemissive $3 \mathrm{D}$ perovskite $\left(\mathrm{CsPCl}_{0.9} \mathrm{Br}_{2.1}\right)$ without solubility problem. Subsequently, PEABr is introduced into the $\mathrm{Cs} \mathrm{PbCl}_{0.9} \mathrm{Br}_{2.1}$ perovskite to passivate the trap states and form quasi-2D perovskite, which helps to obtain a blue-emissive quasi-2D perovskite thin film with PLQY up to $27 \%$. Moreover, we observe a vertically non-uniform distribution of quasi-2D perovskite crystals in the PEDOT:PSS/perovskite hybrid film in which the majority of perovskite crystals disperse on top of the film and only a few are embedded within the PEDOT:PSS layer. Therefore, we modulate the position of the recombination zone to the top of the perovskite film by simply controlling the thickness of the PEDOT:PSS layer to allow the majority of charges recombine in the perovskite emitters. With this strategy, we successfully demonstrate an efficient blue perovskite LED with record EQE of $5.7 \%$ and luminance of $3780 \mathrm{~cd} \mathrm{~m}^{-2}$. The EL peak is located at $480 \mathrm{~nm}$ with FWHM of $21 \mathrm{~nm}$. Our study shows that it is possible to further improve the performance of blue perovskite LEDs by carefully engineering the composition and dimension of the perovskite crystals, as well as the recombination zone position in the devices, paving the way for realization of full-color displays and white-light illumination with perovskite LEDs. However, the lifetimes of our devices are still far from that of commercial LEDs, and it is one of the tough issues has to be solved in perovskite LED field. Our finding indicates that phase separation is probably the main issue causing the device degradation in a mixed-halide perovskite system. Therefore, a pure-halide perovskite system with pure blue and red emission may be ultimately needed for stable perovskite LEDs. In addition, heat produced by the injected current and photon reabsorption may also cause a thermal stability issue (change of film morphology or degradation of perovskite component, etc.). In such case, an efficient device structure design with excellent heat radiation and light out coupling needs to be developed. Moreover, considering the ionic nature of perovskite crystals, the strong electric field in a thin film perovskite LED also could be another possible factor for device degradation, and this would be more severe for a blue perovskite LED as which needs higher applied voltage for operation. The real degradation mechanism of perovskite LEDs is still unclear in this field, and it is of great necessity for an intelligent design for stable perovskite LEDs.

\section{Methods}

Materials and chemicals. $\mathrm{CsBr}$ (99.999\%), $\mathrm{CsCl}$ (99.999\%), and $\mathrm{PbBr}_{2}$ (99.999\%) were purchased from Sigma-Aldrich. PEABr (99.5\%) was purchased from Xi'an Polymer Light Technology Corp. DMSO $(>99.0 \%)$ was purchased from TCI. LiF was purchased from Alfa Aesar. PEDOT:PSS (CH 8000) was purchased from Heraeus. TPBi was purchased from Lumtec. All chemicals were used as received.

Perovskite solution preparation. The $\mathrm{CsPCCl}_{x} \mathrm{Br}_{3-x}$ precursor solutions were prepared by dissolving appropriately stoichiometric $\mathrm{CsCl}, \mathrm{CsBr}$, and $\mathrm{PbBr}_{2}$ in DMSO under continuous stirring for $12 \mathrm{~h}$ at room temperature, keeping the molar concentration of $\mathrm{Pb}^{2+}$ at $0.2 \mathrm{M}$. For the quasi-2D perovskite precursor solution,

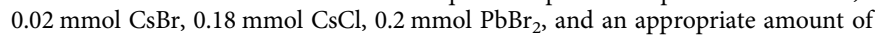
$\mathrm{PEABr}$ were dissolved in $1 \mathrm{~mL}$ DMSO under continuous stirring for $12 \mathrm{~h}$ at room temperature. The ratio of $x \% \mathrm{PEABr}$ refers to the molar ratio between $\mathrm{PEABr}$ and $\mathrm{PbBr}_{2}$ (i.e., $m_{\mathrm{PEABr}} / m_{\mathrm{PbBr} 2}=x \%$ )

Perovskite LED fabrication. The patterned ITO-coated glass substrates were subsequently cleaned by sonication in detergent, deionized water, acetone, and isopropyl alcohol and then dried at $65^{\circ} \mathrm{C}$ in a baking oven. After a 4-min oxygen plasma treatment for ITO, PEDOT:PSS (CH 8000) aqueous solution was spincoated onto the ITO substrate at $3500 \mathrm{rpm}$ for $50 \mathrm{~s}$ and baked at $150{ }^{\circ} \mathrm{C}$ for $15 \mathrm{~min}$ in ambient air. The thickness of the PEDOT:PSS was tuned by diluting the stock solution with different amounts of deionized water. Perovskite precursor solution was then spin-coated onto the PEDOT:PSS film at $2500 \mathrm{rpm}$ for $2 \mathrm{~min}$ in an $\mathrm{N}_{2}$-filled glovebox. Subsequently, the film was annealed at an optimized temperature of $60^{\circ} \mathrm{C}$ for $5 \mathrm{~min}$ (Supplementary Figure 11). Finally, TPBi $(40 \mathrm{~nm})$ and $\mathrm{LiF} / \mathrm{Al}$ electrodes $(1 \mathrm{~nm} / 100 \mathrm{~nm})$ were deposited using a thermal evaporation system under a high vacuum of $<1 \times 10^{-6}$ torr. The device active area was $10 \mathrm{~mm}^{2}$ as defined by the overlapping area of the ITO and Al electrodes. 
Perovskite film and device characterizations. The film thicknesses were determined with a Dektak 150 stylus surface profiling system (Veeco). The ultraviolet-visible absorption spectra were recorded on an HP 8453E spectrophotometer. Steady-state PL was recorded using a Horiba Fluorolog system equipped with a single grating and a monochromatized Xe lamp was used as the excitation source. The crystalline structure of the perovskite films was investigated with an X-ray diffractometer (PANalytical X'pert PRO) equipped with a $\mathrm{Cu}-\mathrm{Ka}$ $\mathrm{X}$-ray tube, using ITO/PEDOT:PSS/perovskite as samples. The SEM images were obtained with a Zeiss EVO 18 SEM based on the sample structure of ITO/PEDOT: PSS/perovskite. The trap densities of the perovskite films were extracted by the dark current-voltage characteristics of the hole-only devices in the device architecture of the ITO/PEDOT:PSS/perovskite/ $\mathrm{MoO}_{3}(10 \mathrm{~nm}) / \mathrm{Ag}(100 \mathrm{~nm})$ through a computer-controlled Keithley 2400 source meter. PLQYs of the perovskite films were recorded by a commercialized PLQY measurement system from Ocean Optics with excitation from a 365-nm LED. Transient PL decay spectra were measured using Quantaurus-Tau fluorescence lifetime measurement system with excitation wavelength of $365 \mathrm{~nm}$ (C11367-03, Hamamatsu Photonics Co., Japan). UPS spectra were collected on Thermo Fisher Scientific Escalab 250 Xi equipment with an applied bias of $-5 \mathrm{~V}$. A He I ultraviolet radiation source $(21.22 \mathrm{eV})$ was used. The pressure in the analysis chamber during measurement was about $5 \times 10^{-10}$ mbar. The energy scale was based on the Fermi edge of gold $(0 \mathrm{eV})$. The crosssectional STEM images and EDS measurement were taken with a FIB-TEM Helios Nanolab 450S. The atomic force microscope measurements were carried out using a Digital Instrumental Multimode Nanoscope IIIa in tapping mode. Capacitance-voltage measurements were carried out with a Paios 4.0 Measurement Instrument (FLUXiM AG, Switzerland). The I-V-L curve, EL spectrum, EQE, and operating lifetime of the perovskite LED were recorded simultaneously by a commercialized system (XPQY-EQE-350-1100, Guangzhou Xi Pu Optoelectronics Technology Co., Ltd.) that was equipped with an integrated sphere (GPS-4P-SL, Labsphere) and a photodetector array (S7031-1006, Hamamatsu Photonics). All of the device characterization tests of the perovskite LEDs were carried out in an $\mathrm{N}_{2}-$ filled glovebox.

\section{Data availability}

The data that support the findings of this study are available from the corresponding author upon reasonable request.

Received: 6 October 2018 Accepted: 12 February 2019

Published online: 04 March 2019

\section{References}

1. Luo, D. et al. Enhanced photovoltage for inverted planar heterojunction perovskite solar cells. Science 360, 1442-1446 (2018).

2. Chen, Z. et al. Recombination dynamics study on nanostructured perovskite light-emitting devices. Adv. Mater. 30, 1801370 (2018).

3. Sun, H., Tian, W., Cao, F., Xiong, J. \& Li, L. Ultrahigh-performance selfpowered flexible double-twisted fibrous broadband perovskite photodetector. Adv. Mater. 30, 1706986 (2018).

4. Wang, Y. et al. All-inorganic colloidal perovskite quantum dots: a new class of lasing materials with favorable characteristics. Adv. Mater. 27, 7101-7108 (2015).

5. Wolf, S. D. et al. Organometallic halide perovskites: sharp optical absorption edge and its relation to photovoltaic performance. J. Phys. Chem. Lett. 5, 1035-1039 (2014).

6. Stranks, S. D. et al. Electron-hole diffusion lengths exceeding 1 micrometer in an organometal trihalide perovskite absorber. Science 342, 341-344 (2013).

7. Braly, I. L. et al. Hybrid perovskite films approaching the radiative limit with over $90 \%$ photoluminescence quantum efficiency. Nat. Photon. 12, 355-361 (2018).

8. Akkerman, Q. A., Rainò, G., Kovalenko, M. V. \& Manna, L. Genesis, challenges and opportunities for colloidal lead halide perovskite nanocrystals. Nat. Mater. 17, 394-405 (2018).

9. Cho, H. et al. Overcoming the electroluminescence efficiency limitations of perovskite light-emitting diodes. Science 350, 1222-1225 (2015).

10. Song, J. et al. Quantum dot light-emitting diodes based on inorganic perovskite cesium lead halides $\left(\mathrm{CsPbX}_{3}\right)$. Adv. Mater. 27, 7162-7167 (2015).

11. Protesescu, L. et al. Nanocrystals of cesium lead halide perovskites $\left(\mathrm{CsPbX}_{3}\right.$, $\mathrm{X}=\mathrm{Cl}, \mathrm{Br}$, and I): novel optoelectronic materials showing bright emission with wide color gamut. Nano Lett. 15, 3692-3696 (2015).

12. Akkerman, Q. A. et al. Tuning the optical properties of cesium lead halide perovskite nanocrystals by anion exchange reactions. J. Am. Chem. Soc. 137, 10276-10281 (2015)

13. Xiao, Z. et al. Efficient perovskite light-emitting diodes featuring nanometresized crystallites. Nat. Photon. 11, 108-115 (2017).

14. Yuan, M. et al. Perovskite energy funnels for efficient light-emitting diodes. Nat. Nanotechnol. 11, 872-877 (2016).
15. Yang, X. et al. Efficient green light-emitting diodes based on quasi-twodimensional composition and phase engineered perovskite with surface passivation. Nat. Commun. 9, 570 (2018).

16. Cao, Y. et al. Perovskite light-emitting diodes based on spontaneously formed submicrometre-scale structures. Nature 562, 249-253 (2018).

17. Lin, K. et al. Perovskite light-emitting diodes with external quantum efficiency exceeding 20 per cent. Nature 562, 245-248 (2018).

18. Chiba, T. et al. Anion-exchange red perovskite quantum dots with ammonium iodine salts for highly efficient light-emitting devices. Nat. Photon. 12, 681-687 (2018)

19. Chen, Z. et al. High-performance color-tunable perovskite light emitting devices through structural modulation from bulk to layered film. Adv. Mater. 29, 1603157 (2017).

20. Wang, Q., Ren, J., Peng, X.-F., Ji, X.-X. \& Yang, X.-H. Efficient sky-blue perovskite light-emitting devices based on ethylammonium bromide induced layered perovskites. ACS Appl. Mater. Interfaces 9, 29901-29906 (2017).

21. Tang, X. et al. Local observation of phase segregation in mixed-halide perovskite. Nano Lett. 18, 2172-2178 (2018).

22. Vashishtha, P. \& Halpert, J. E. Field-driven ion migration and color instability in red-emitting mixed halide perovskite nanocrystal light-emitting diodes. Chem. Mater. 29, 5965-5973 (2017)

23. Saidaminov, M. I. et al. Suppression of atomic vacancies via incorporation of isovalent small ions to increase the stability of halide perovskite solar cells in ambient air. Nat. Energy 3, 648-654 (2018).

24. Leijtens, T. et al. Towards enabling stable lead halide perovskite solar cells; interplay between structural, environmental, and thermal stability. J. Mater. Chem. A 5, 11483-11500 (2017).

25. Shang, Y., Li, G., Liu, W. \& Ning, Z. Quasi-2D inorganic $\mathrm{CsPbBr}_{3}$ perovskite for efficient and stable light-emitting diodes. Adv. Funct. Mater. 28, 1801193 (2018).

26. Chen, P. et al. Charge-transfer versus energy-transfer in quasi-2D perovskite light-emitting diodes. Nano Energy 50, 615-622 (2018).

27. Chang, J. et al. Enhanced performance of red perovskite light-emitting diodes through the dimensional tailoring of perovskite multiple quantum wells. J. Phys. Chem. Lett. 9, 881-886 (2018).

28. Kumawat, N. K. et al. Band gap tuning of $\mathrm{CH}_{3} \mathrm{NH}_{3} \mathrm{~Pb}\left(\mathrm{Br}_{1-x} \mathrm{Cl}_{x}\right)_{3}$ hybrid perovskite for blue electroluminescence. ACS Appl. Mater. Interfaces 7, 13119-13124 (2015)

29. Yan, F. et al. Highly efficient visible colloidal lead-halide perovskite nanocrystal light-emitting diodes. Nano Lett. 18, 3157-3164 (2018).

30. Zhang, X. et al. Stable high efficiency two-dimensional perovskite solar cells via cesium doping. Energy Environ. Sci. 10, 2095-2102 (2017).

31. Yu, M. et al. Control of barrier width in perovskite multiple quantum wells for high performance green light-emitting diodes. Adv. Optical Mater https://doi.org/10.1002/adom.201801575 (2018)

32. Xing, J. et al. Color-stable highly luminescent sky-blue perovskite lightemitting diodes. Nat. Commun. 9, 3541 (2018).

33. Chen, Z. et al. Stable $\mathrm{Sn} / \mathrm{Pb}$-based perovskite solar cells with a coherent $2 \mathrm{D} / 3 \mathrm{D}$ interface. iScience 9, 337-346 (2018).

34. Xue, Q. et al. Metallohalide perovskite-polymer composite film for hybrid planar heterojunction solar cells. RSC Adv. 5, 775-783 (2015).

35. Wang, J. et al. All-solution-processed pure formamidinium-based perovskite light-emitting diodes. Adv. Mater. 30, 1804137 (2018).

36. Wang, N. et al. Perovskite light-emitting diodes based on solutionprocessed self-organized multiple quantum wells. Nat. Photon. 10, 699-704 (2016).

37. Xu, J. et al. Highly efficient inverted blue light-emitting diodes by thermal annealing and interfacial modification. Org. Electron. 49, 1-8 (2017).

38. Li, W. et al. Extremely low color-temperature white organic electroluminescence devices based on the control of exciton recombination zone. Phys. Stat. Sol. A 213, 2400-2405 (2016).

39. Bhansali, U. S. et al. Controlling the carrier recombination zone for improved color stability in a two-dopant fluorophore/phosphor white organic lightemitting diode. Appl. Phys. Lett. 94, 203501 (2009).

40. Li, G. et al. Highly efficient perovskite nanocrystal light-emitting diodes enabled by a universal crosslinking method. Adv. Mater. 28, 3528-3534 (2016)

41. Deng, W. et al. Organometal halide perovskite quantum dot light-emitting diodes. Adv. Funct. Mater. 26, 4797-4802 (2016).

42. Wang, Z., Cheng, T., Wang, F., Dai, S. \& Tan, Z. Morphology engineering for high-performance and multicolored perovskite light-emitting diodes with simple device structures. Small 12, 4412-4420 (2016).

43. Pan, J. et al. Highly efficient perovskite-quantum-dot light-emitting diodes by surface engineering. Adv. Mater. 28, 8718-8725 (2016).

44. Hu, H., Salim, T., Chen, B. \& Lam, Y. M. Molecularly engineered organic-inorganic hybrid perovskite with multiple quantum well structure for multicolored light-emitting diodes. Sci. Rep. 6, 33546 (2016)

45. Zhang, X. et al. Bright perovskite nanocrystal films for efficient light-emitting devices. J. Phys. Chem. Lett. 7, 4602-4610 (2016). 
46. Cheng, L. et al. Sky-blue perovskite light-emitting diodes based on quasi-twodimensional layered perovskites. Chin. Chem. Lett. 28, 29-31 (2017).

47. Kim, H. P. et al. High-efficiency, blue, green, and near-infrared light-emitting diodes based on triple cation perovskite. Adv. Opt. Mater. 5, 1600920 (2017).

48. Yao, E.-P. et al. High-brightness blue and white LEDs based on inorganic perovskite nanocrystals and their composites. Adv. Mater. 29, 1606859 (2017).

49. Gangishetty, M. K., Hou, S., Quan, Q. \& Congreve, D. N. Reducing architecture limitations for efficient blue perovskite light-emitting diodes. $A d v$. Mater. 30, 1706226 (2018)

50. Yang, D. et al. Large-scale synthesis of ultrathin cesium lead bromide perovskite nanoplates with precisely tunable dimensions and their application in blue light-emitting diodes. Nano Energy 47, 235-242 (2018).

51. Hou, S., Gangishetty, M. K., Quan, Q. \& Congreve, D. N. Efficient blue and white perovskite light-emitting diodes via manganese doping. Joule $\mathbf{2}$ 2421-2433 (2018).

\section{Acknowledgements}

This study was financially supported by the Ministry of Science and Technology of the People's Republic of China (No. 2017YFA0206600), Science and Technology Program of Guangzhou, China (No. 201607020010), and National Natural Science Foundation of China (Nos. 21761132001, 51573057, and 91733302).

\section{Author contributions}

Z.L. fabricated the perovskite LEDs and carried out the device characterizations. Z.L., Z.C., Y.Y., and Q.X. carried out the perovskite film characterizations. Z.C., Z.L., and H.-L.Y. analyzed the data and wrote the manuscript. Z.C. and H.-L.Y. led the project. H.-L.Y. and Y.C. supervised the research.

\section{Additional information}

Supplementary Information accompanies this paper at https://doi.org/10.1038/s41467019-09011-5.

Competing interests: The authors declare no competing interests.

Reprints and permission information is available online at http://npg.nature.com/ reprintsandpermissions/

Journal peer review information: Nature Communications thanks the anonymous reviewers for their contribution to the peer review of this work.

Publisher's note: Springer Nature remains neutral with regard to jurisdictional claims in published maps and institutional affiliations.

\begin{abstract}
cc (i) Open Access This article is licensed under a Creative Commons Attribution 4.0 International License, which permits use, sharing, adaptation, distribution and reproduction in any medium or format, as long as you give appropriate credit to the original author(s) and the source, provide a link to the Creative Commons license, and indicate if changes were made. The images or other third party material in this article are included in the article's Creative Commons license, unless indicated otherwise in a credit line to the material. If material is not included in the article's Creative Commons license and your intended use is not permitted by statutory regulation or exceeds the permitted use, you will need to obtain permission directly from the copyright holder. To view a copy of this license, visit http://creativecommons.org/ licenses/by/4.0/.
\end{abstract}

(C) The Author(s) 2019 